\title{
LAS INVESTIGACIONES CORRELACIONALES DE LAS CIENCIAS SOCIALES EN TORNO A LAS PROBLEMÁTICAS HIDROLÓGICAS
}

\section{THE CORRELATIONAL INVESTIGATIONS OF PROBLEMATIC SOCIAL ISCIENCES AROUND THE HYDROLOGIC ONES}

\author{
Cruz García Lirios \\ Universidad Autónoma Metropolitana de México (México) \\ csh96327267@titlani.uam.mx
}

Recibido/Received: 04/06/09

Aceptado/Accepted:03/09/09

\section{RESUMEN}

Se exponen las correlaciones entre las variables valorativas, evaluativas, perceptuales, actitudinales, intencionales y conductuales en torno a las problemáticas hidrológicas de escasez, desabasto e insalubridad. A partir de una categorización de las mencionadas correlaciones en exógenas para las variables culturales y endógenas para las variables situacionales, se infieren los modelos que explicaran la influencia de las estructuras sociales en los comportamientos individuales.

PALABRAS CLAVE

Estructura, modelo, variable y correlación exógena-endógena.

\section{ABSTRACT}

The correlations between valuing, evaluative, perceptual, attitudinal, intentional and behavioral the variables around problematic the hydrologic ones of shortage, and unhealthiness are exposed. From a categorization of the mentioned correlations in exogenous for the cultural and endogenous variables for the situational variables, the models are inferred that explained the influence of the social structures in the individual behaviors.

\section{KEYWORDS}

Structure, model, variable and exogenous-endogenous correlation.

\section{INTRODUCCIÓN}

La correlación bivariada estadística estimada con el parámetro " $\mathrm{r}$ " de Pearson permite una abstracción de las relaciones concretas asociadas en una variable $\mathrm{X}$ y una variable $\mathrm{Y}$. La fuerza de asociación entre una variable $\mathrm{X}$ y otra variable $\mathrm{Y}$ es conocida como correlación. Se trata de un análisis en el que se establecen las relaciones asociadas entre las variables que configuran una estructura cultural, social, comunitaria, económica, política, institucional, corporativa, educativa o familiar. Dicha estructura es evidenciada en un modelo en el que las variables y los constructos explican la influencia de la estructura en los individuos. Dicho modelo, se construye a partir de las relaciones asociadas entre las variables para inferir sus relaciones causales. En torno a la escasez de agua, las 
asociaciones exógenas y endógenas entre los valores, las creencias, las percepciones, las actitudes, las habilidades y las intenciones, orientan su modelización como determinantes del dispendio o ahorro de agua.

\section{ASOCIACIONES EXÓGENAS ${ }^{1}$}

Son las relaciones entre las variables culturales (valores) y las variables ideológicas (creencias). Es decir, se considera que tanto la cultura como la ideología influyen en el individuo a través de los valores y las creencias que se amplifican en los discursos de la gente y que el individuo capta, aprende y reproduce ante una situación específica. En este sentido, el abasto irregular de agua característico de las ciudades modernas y las urbes periféricas, está asociado a valores y creencias en torno a su disponibilidad exclusiva para el consumo humano o su disponibilidad compartida entre las especies.

\section{1. "Las asociaciones endógenas desvelan las diferencias intra e inter culturales e ideológicas"}

Las sociedades colectivistas tales como las asiáticas, latinas y europeas del este se caracterizan por valores biosféricos-altruistas y creencias ecocéntricas que favorecen el cuidado del medio ambiente al ser considerado como su hábitat y las especies como sus compañeras hermanas de coexistencia. En torno a la insalubridad por la infraestructura hidrológica deficiente o inexistente, las comunidades y los barrios populares se solidarizan para el auto cuidado de los niños. Esto significa que en la medida en que la insalubridad aumenta, la solidaridad comunitaria también se incrementa.

Las sociedades europeas y norteamericanas, en contraste, se caracterizan por valores individualistas y creencias antropocéntricas. Incluso en los grupos migrantes colectivistas que residen en estas sociedades se observa un cambio de valores y creencias que los acerca a individualismo y al antropocentrismo. La disponibilidad de agua, asociada a los valores de sobre-explotación y las creencias de abundancia del recurso, orientan la elaboración de un modelo en el que se evidencien el aumento de las dos variables culturales e ideológicas en la medida en que se incrementa la información sobre la abundancia de agua.

La influencia de la estructura social individualista y antropocéntrica también se observa en los países con economías emergentes (Brasil, Rusia, India, China) que serán desarrolladas en la década que se aproxima. Se trata de economías que transitan del colectivismo al individualismo, del biosferismo al industrialismo, del ecocentrismo al antropocentrismo. El crecimiento económico está asociado con la inversión pública en infraestructura hidrológica. Los proyectos energéticos e hidrológicos están correlacionados con las necesidades de las urbes. La inversión en torno al servicio público del agua está asociado al incremento de la población en las ciudades, sus dimensiones, servicios y migración. El consumo de agua registra un incremento en sus tarifas asociadas con la escasez en los barrios periféricos. El desabasto de agua vinculada con la insalubridad e 
implicada con las epidemias aumenta las muertes infantiles.

\section{ASOCIACIONES ENDÓGENAS ${ }^{2}$}

Son las relaciones entre las variables espaciales (diseños), las variables económicas (riesgo y utilidad), las variables educativas (conocimientos) y las variables individuales (actitudes, habilidades, intenciones, comportamientos). Los estudios en torno a la influencia de las edificaciones sobre la percepción individual demostraron que la estética, la funcionalidad y el diseño tienen un efecto directo, positivo y significativo sobre la satisfacción del cliente. Posteriormente, los estudios en torno a la influencia de las masas en el interior de las edificaciones sobre el comportamiento humano demostraron que el hacinamiento, el ruido o la densidad son factores que determinan el estrés del cliente. Finalmente, los estudios en torno a la influencia de los eventos en el interior de las edificaciones sobre la cognición individual demostraron que las personas se forman actitudes hacia los eventos, edificios y espectadores. Las edificaciones vinculadas con la bioseguridad hidrológica (reservas de agua potable) demuestran la relevancia de las políticas sanitarias, las contingencias epidémicas, las catástrofes pandémicas, la competencia por los recursos y la solidaridad comunitaria.

\section{1. "Las asociaciones endógenas desvelan impactos diferentes de los espacios, los ingresos, los conocimientos y las actitudes sobre los comportamientos"}

En las economías industriales con políticas neoliberales, los comportamientos contaminantes han sido asociados con las percepciones utilitarias, las actitudes racionales y los conocimientos tecnológicos. Los proyectos hidrológicos están diseñados para incrementar la utilidad personal más que la utilidad social. Es decir, el servicio de agua potable sólo está disponible para aquellas zonas que pueden pagar el costo del servicio.

En las economías postindustriales con políticas sociales, los comportamientos preservadores han sido vinculados con las percepciones de riesgo, las actitudes afectivas y los conocimientos sociales. Los proyectos hidrológicos están vinculados a los servicios de todo tipo. Se trata de abastecer a las zonas comerciales relacionadas con el turismo. En la medida en que se incrementa el turismo, la disponibilidad del agua aumenta en dichas zonas comerciales.

En las economías informacionales con políticas sostenibles, los comportamientos ecológicos han sido vinculadas con las percepciones de responsabilidad, las actitudes globales y los conocimientos organizacionales. Los proyectos hidrológicos se enlazan con la normatividad sostenible que obliga una disponibilidad hidrológica equitativa entre las zonas y las especies.

A partir de los estudios asociativos, tanto exógenos como endógenos, se han abstraído estructuras económicas, políticas y sociales que influyen en los individuos. Los análisis de correlación evidencian los modelos de consumo que culpan a los individuos del deterioro global y plantean el accionar aislado como la solución al problema global. Ante la escasez, desabasto e insalubridad hidrológicas se plantea que la educación ambiental es la acción indicada para prevenir dichas problemáticas y las ecotasas (multas e incentivos) 
son las estrategias fiscales efectivas para el desarrollo sostenible.

Las asociaciones, tanto exógenas como endógenas, orientan el diseño de modelos teórico-estructurales. Una relación causal entre una variable $\mathrm{X}$ y una variable $\mathrm{Y}$ subyace de una asociación exógena entre una variable $\mathrm{W}$ y una variable $\mathrm{X}$. $\mathrm{O}$ bien, los determinantes de una variable $\mathrm{Z}$ subyacen de las asociaciones entre $\mathrm{W}, \mathrm{X}$ y $\mathrm{Y}$. Es decir, a partir de las asociaciones se infieren las relaciones causales. Si existe una asociación significativa entre las variables independientes pueden haber relaciones causales entre ellas. Si existen asociaciones espurias entre las variables independientes pueden haber relaciones causales con una variable dependiente. Una correlación positiva y significativa entre la escasez, el desabasto y la insalubridad hidrológicas permite la elaboración de un modelo en el que el ahorro de agua está determinado por las tres situaciones hidrológicas. Una correlación negativa y significativa entre las tres variables permite un diseño en el que el dispendio de agua es el efecto esperado. Una correlación espuria entre las tres situaciones hidrológicas orienta el diseño de un modelo en el que otras variables situaciones estarían explicando el dispendio o el ahorro de agua.

\section{NOTAS}

${ }^{1}$ Amérigo, Aragonés, Sevillano y Cortés (2005) con una muestra de 212 estudiantes españoles establecieron la correlación positiva y significativa $(r=.32 ; \mathrm{p}<.01)$ entre el egocentrismo y el biosferismo, dos dimensiones, de los valores ambientales.

Bechtel, Asai, Corral y González (2006) con una muestra de 1.358 residentes del mundo (200 japoneses, 308 estadounidenses, 450 mexicanos y 400 peruanos) demostraron la relación directa, positiva y significativa entre tres variables ideológicas. En Japón encontraron que las creencias en torno a un equilibrio entre las necesidades de la humanidad y las especies restantes está relacionada con las creencias referentes al crecimiento limitado de la humanidad $(r=.41 ; p<.05)$. En México establecieron la relación positiva entre las creencias que sostienen la excepcionalidad de los humanos ante las leyes de la naturaleza y los limites al crecimiento $(\mathrm{r}=.17 ; \mathrm{p}<.05)$ y esta última variable con las creencias de equilibrio entre las necesidades humanas y las de la naturaleza $(\mathrm{r}=.42 ; \mathrm{p}<.05)$. En Perú encontraron que los límites al crecimiento están relacionados con el balance entre la naturaleza y la humanidad $(\mathrm{r}=.84)$. En contraste, Tanto en Japón como en Perú las creencias sobre la excepcionalidad de la humanidad están relacionadas negativamente con las creencias de equilibrio ( $\mathrm{r}-.62 ; \mathrm{p}<.05$ en Japón y $\mathrm{r}-.38 ; \mathrm{p}<.05$ en Perú). Finalmente, en este último país, la excepcionalidad humana y los límites al crecimiento están inversamente relacionados $(\mathrm{r}-.43 ; \mathrm{p}<.05)$

Pato, Tróccoli y Tamayo (2002) con una muestra de 281 estudiantes brasileños establecieron la relación concomitante entre las variables universalismo y seguridad $(\mathrm{r}=.30 ; \mathrm{p}<.01)$

Schultz, Gouveia, Cameron, Tankha, Schmuck y Franek (2005) con una muestra de 720 residentes mundiales demostraron las relaciones positivas y significativas entre los valores altruistas con los valores trascendentes $(\mathrm{r}=.41 ; \mathrm{p}<.01)$ en participantes rusos, los valores trascendentes con los valores biosféricos $(\mathrm{r}=.33 ; \mathrm{p}<.01)$ en participantes brasileños, los valores trascendentes con los valores biosféricos $(\mathrm{r}=.35$; $\mathrm{p}<.01$ ) en participantes checos. En contraste, demostraron las relaciones negativas y significativas entre los valores altruistas con los valores individualistas $(r=-.48 ; \mathrm{p}<.01)$ en participantes rusos, los valores biosféricos con los valores individualistas $(\mathrm{r}=-.31 ; \mathrm{p}<.01)$ en participantes checos. Sin embargo, demostraron las relaciones negativas y significativas entre los valores altruistas con los valores conservacionistas $(r=-.22 ; \mathrm{p}<.05)$ en participantes rusos, los valores biosféricos con los valores conservacionistas $(\mathrm{r}=-.21 ; \mathrm{p}<.05)$ en participantes brasileños y la relación positiva y significativa entre los valores egoístas con los valores conservacionistas $(r=.21 ; \mathrm{p}<.05)$ en participantes checos. 
${ }^{2}$ Martínportugués, Gallego y Ruiz (2006) con una muestra de 225 residentes españoles demostraron tanto las correlaciones positivas $(\mathrm{r}=.46 \mathrm{y} \mathrm{r}=.32 ; \mathrm{p}<.01)$ como negativas $(\mathrm{r}=-.28 \mathrm{y} \mathrm{r}=-.31 ; \mathrm{p}<.01)$ de la percepción ambiental con la satisfacción residencial, la satisfacción ambiental, la percepción ruidosa y las molestias sonoras respectivamente. Demostraron las correlaciones positivas $(r=37$ y $r=.37 ; \mathrm{p}<.01)$ y negativas $(\mathrm{r}=-.22 ; \mathrm{p}<.01)$ de la satisfacción residencial con la satisfacción barrial, la satisfacción ambiental y la percepción ruidosa respectivamente.

Salazar, Hernández, Martín y Hess (2006) con una muestra de 196 estudiantes españoles demostraron la relación positiva y significativa entre tres variables socio-espaciales; el tipo de construcción estuvo relacionado con los efectos ambientales $(r=.34 ; \mathrm{p}<.05)$ y los efectos sociales $(.32 ; \mathrm{p}<.05)$.

Acosta y Montero (2001) con una muestra de 30 estudiantes en la ciudad de México, demostraron que la conducta ambiental responsable está asociada $(\mathrm{r}=.45 ; \mathrm{p}<.05)$ con el índice de habilidades y conocimientos de la acción ambiental.

Bustos, Flores y Andrade (2004) con una muestra de 349 residentes mexicanos demostraron la relación directa, positiva y significativa entre tres variables conductuales proambientales; lavado de baños con aseo personal $(\mathrm{r}=.17 ; \mathrm{p}<01)$, aseo de dientes con baño personal $(\mathrm{r}=.18 ; \mathrm{p}<.01)$. La relación entre cuatro variables conservacionistas; creencias de obligación de cuidar el agua con habilidades instrumentales de ahorro $(r=.24 ; \mathrm{p} .01)$, conocimientos sobre el manejo de agua con motivos de protección socio-ambiental $(\mathrm{r}=.42 ; \mathrm{p}<.01)$ y habilidades instrumentales de ahorro con motivos de protección socio-ambiental $(\mathrm{r}=.27 ; \mathrm{p}<.01)$. la relación entre siete factores proambientales; creencias de obligación de cuidar el agua con locus de control interno $(\mathrm{r}=.43 ; \mathrm{p}<.01)$, con el ahorro de agua $(\mathrm{r}=.45$; $\mathrm{p}<.01)$, con los motivos de protección ambiental $(\mathrm{r}=.24 ; \mathrm{p}<.01)$, con la percepción de riesgos para la salud $(\mathrm{r}=.26 ; \mathrm{p}<.01)$, a su vez la percepción esta relacionada con el locus de control interno $(\mathrm{r}=.30 ; \mathrm{p}<$ $.01)$, con el comportamiento proambiental $(\mathrm{r}=.23 ; \mathrm{p}<.01)$ y con los motivos de protección ambiental $(\mathrm{r}$ $=.27)$ los cuales a su vez están relacionados con el locus de control $(\mathrm{r}=.28 ; \mathrm{p}<.01)$, con el ahorro de agua $(\mathrm{r}=.12 ; \mathrm{p}<.01)$ y con las habilidades instrumentales $(\mathrm{r}=.42)$ los que a su vez están relacionados con el locus de control $(\mathrm{r}=.38 ; \mathrm{p}<.01)$ y el ahorro de agua $(\mathrm{r}=.33 ; \mathrm{p}<.01)$. Finalmente, los conocimientos del manejo de agua están asociados con el locus de control $(r=.16 ; \mathrm{p}<.01)$ y con el comportamiento proambiental $(\mathrm{r}=.47 ; \mathrm{p}<.01)$

Corral, Frías, Fraijó y Tapia (2006) con una muestra de 150 habitantes mexicanos demostraron la relación directa, positiva y significativa entre cinco variables conductuales; tendencia al riesgo con el comportamiento antiambiental $(\mathrm{r}=.51 ; \mathrm{p}<.05)$, la conducta antisocial $(\mathrm{r}=.68 ; \mathrm{p}<.05)$ y la falta de autocontrol $(\mathrm{r}=.48 ; \mathrm{p}<.05)$. A su vez esta última variable se relacionó con la conducta antisocial $(\mathrm{r}=.27$; $\mathrm{p}<.05)$ y la conducta antisocial $(\mathrm{r}=.45 ; \mathrm{p}<.05)$. Tanto el comportamiento antiambiental como antisocial tuvieron una relación similar $(\mathrm{r}=.84 ; \mathrm{p}<.05)$. En contraste, las relaciones directas, negativas y significativas se establecieron entre el comportamiento proambiental frente a la tendencia al riesgo $(\mathrm{r}=$ $.12 ; \mathrm{p}<.05)$ y la falta de autocontrol $(\mathrm{r}=-.19)$.

Meinhold \& Malkus (2005) con una muestra de 848 norteamericanos correlacionaron la auto eficacia con las actitudes ambientales $(\mathrm{r}=.23)$, con los conocimientos ambientales $(\mathrm{r}=.16)$ y con el comportamiento pro ambiental $(\mathrm{r}=.30)$, las actitudes ambientales con los conocimientos ambientales $(\mathrm{r}=.18)$ y con el comportamiento proambiental $(\mathrm{r}=.45)$, los conocimientos ambientales con el comportamiento pro ambiental $(\mathrm{r}=.34)$ todos con una significancia menor a .001

\section{BIBLIOGRAFÍA}

ACOSTA, J. y MONTERO, M. (2001): "Relación entre conducta proambiental y algunos componentes psicológicos en estudiantes mexicanos”, Medio Ambiente y Comportamiento Humano, 2, 45-58.

AMÉRIGO, M. (2006): "La investigación en España sobre actitudes proambientales y comportamiento ecológico", Medio Ambiente y Comportamiento Humano, 7, 45-75

AMÉRIGO, M.; ARAGONÉS, J.; SEVILLANO, V. y CORTÉS B. (2005): "La estructura de las creencias sobre la problemática medioambiental", Psicothema, 17, 257-262 
AMÉRIGO, M. y GONZÁLEZ, A. (2001): "Los valores y las creencias medio ambientales en relación con las decisiones sobre dilemas ecológicos", Estudios de Psicología, 22, 65-73.

BECHTEL, R.; ASAI, M.: CORRAL, V. y GONZÁLEZ, A. (2006): "A cross cultural study of environmental beliefs structures in USA, Japan, México and Peru", International Journal of Psychology, $41,145-151$

BECHTEL, R.; CORRAL, V. y PINHEIRO, J. (1999): "Environmental belief systems United Status", Brazil and México, Journal of Cross-Cultural Psychology, 30, 122-128

BUSTOS, J. (2004): Modelo de conducta proambiental para el estudio de la conservación de agua potable, Tesis de Doctorado, Facultad de Psicología, Universidad Nacional Autónoma de México.

BUSTOS, J.; FLORES, M. y ANDRADE, P. (2000): "Evaluación de problemas ambientales, calidad del ambiente y creencias de afectación de la salud", La Psicología Social en México, 8, 445-451.

- (2004): "Predicción de la conservación de agua a partir de factores socio cognitivos", Medio Ambiente y Comportamiento Humano. 5, 53-70

BUSTOS, J.; FLORES, L.; BARRIENTOS, C. y MARTÍNEZ, J. (2004): “Ayudando a contrarrestar el deterioro ecológico: atribución y motivos para conservar agua”. La Psicología Social en México. 10, 521 526

BUSTOS, J.; MONTERO, M. y FLORES, L. (2002): “Tres diseños de intervención antecedente para promover conducta protectora del ambiente", Medio Ambiente y Comportamiento Humano, 3, 63-88.

CORRAL, V. (2003a): ¿Mapas cognitivos o conductas ambientales? en DÍAZ, E., y ANAYA, M. (comp.), Perspectivas sobre el cognitivismo en psicología, (pp. 37-79), México, UNAM (Iztacala).

CORRAL, V. (2003b): "Determinantes psicológicos o situacionais do comportamento de conservaçào de àgua: um modelo estructural”, Estudos de Psicología. 8, 245-252.

CORRAL, V. (2002a): “A structural model of proenvironmental competency", Environment \& Behavior, 34, 531-549.

CORRAL, V. (2002b): Avances y limitaciones en la medición del comportamiento proambiental, en Guevara, J. y Mercado, S. (coords.) Temas Selectos de Psicología Ambiental, (pp. 483-510), México, UNAM-GRECO-UNILIBRE.

CORRAL, V. (2002c): Structural Equation Modelling. in Bechtel, R. y Churcman, A. (eds.) Handbook of Environmental Psychology, (pp. 256-270), New York, Wiley \& Sons, Inc.

CORRAL, V. (1998). Interacciones ambiente / conducta: algunas áreas de investigación. En Alcaraz, V.M. y Bouzas, A. (coords.), Las aportaciones mexicanas a la psicología, (pp. 55-70), México, UNAM.

CORRAL, V.; BECHTEL, R.; ARMENDÁRIZ, L. I. y ESQUER, A. B. (1997): "La estructura de las creencias ambientales en universitarios mexicanos: el Nuevo Paradigma Ambiental", Revista Mexicana de Psicología, 14, 173-181.

CORRAL, V.; BECHTEL, R. y FRAIJÓ, B. (2003): "Environmental beliefs and water conservation: an empirical study", Journal of Environmental Psychology, 23, 247-257.

CORRAL, V.; CAPDEVIELLE, F.; GARIBALDI, L. y ENCINAS, L. (1986): "Estrategias conceptuales para la reducción de consumo doméstico de agua en una zona urbana”, La Psicología Social en México, $1,475-479$.

CORRAL, V. y ENCINAS, L. (2001): "Variables disposicionales, situacionales y demográficas en el reciclaje de metal y papel", Medio Ambiente y Comportamiento Humano, 2, 1-19.

CORRAL, V.; FRAIJO, B.; FRÍAS, M.; GONZÁLEZ, D. y PINHEIRO, J. (2004): "Propensión al presente, al pasado y al futuro y sus relaciones con el ahorro de agua", La Psicología Social en México, $10,547-552$

CORRAL, V y FRÍAS, M. (2006): "Personal normative beliefs, antisocial behaviour, and residential water conservation", Environment and Behavior, 38, 406-421

CORRAL, V., FRÍAS, M. y GONZÁLEZ, D. (2001): "On the relationship between antisocial and antienvironmental behaviors: an empirical study”, Population and Environment, 24, 273-286.

CORRAL, V.; GARIBALDI, L. y ENCINAS, L. (1987): "Estudio exploratorio de patrones conductuales de consumo doméstico de agua en zona urbana", Revista Sonorense de Psicología, 2, 87-93.

CORRAL, V. y OBREGÓN, F. J. (1992): "Modelos predictores del comportamiento proambientalista", Revista Sonorense de Psicología, 6, 5-14. 
CORRAL, V. y PINHEIRO, J. (1999): "Condiçoes para o estudo do comportamento próambiental", Estudos de Psicología, 4, 7-22.

CORRAL, V. y ZARAGOZA, F. (2000): "Bases sociodemográficas y psicológicas de la conducta de reutilización: Un Modelo estructural", Medio Ambiente y Comportamiento Humano, 1, 9-29.

CORRAL, V.; VARELA, C. y GONZÁLEZ, D. (2002): "Una taxonomía funcional de competencias proambientales", La Psicología Social en México, 9, 592-597.

GOUVEIA, V.; MARTÍNEZ, E.; MEIRA, M. y LEMOS, T. (2001): "A estructura e o conteúdo universais dos valores humanos: analise fatorial confirmatória da tipología de Schwartz", Estudos de Psicología, 6, 133-142

MARTÍN PORTUGUÉS, C.; GALLEGO, J. y RUIZ, D. (2006): "Efectos del ruido comunitario", Revista de Acústica, 34, 31-39

MILFONT, T. y GOUVEIA, V. (2006): "Time perspective and values: an exploratory study of their relations to environmental attitudes", Journal of Environmental Psychology, 26, 72-82

PATO, C. y TAMAYO, A. (2006): "Valores, creencias ambientales y comportamiento ecológico de activismo", Medio Ambiente y Comportamiento Humano, 7, 51-66

PATO, C.; TRÓCCOLI, B. y TAMAYO, A. (2002): "Values and ecological behavior: an empirical study of Brazilians students", $17^{\text {th }}$ Conference of IAPS 2002.

SALAZAR, M.; HERNÁNDEZ, B.; MARTÍN, A. y HESS, S. (2006): "Predictores de la asignación de castigo en transgresores de las leyes medio ambientales", Medio Ambiente y Comportamiento Humano, $7,105-122$

\section{Breve currículo:}

\section{Cruz García Lirios}

Licenciado en Psicología Social por la Universidad Autónoma Metropolitana en México. Realizó una Maestría en Trabajo Social en la Escuela Nacional de Trabajo Social en la Universidad Nacional Autónoma de México, obtuvo este segundo título con su proyecto de tesis: "La exclusión hidrológica en la delegación Iztapalapa de la Ciudad de México" asesorado y evaluado a través de un comité integrado por cinco doctores especialistas en esta área. Participó en el proyecto de investigación "Un Equipo contra el Sida" en el Instituto Mexicano de Investigación de Familia y Población. Dicho proyecto estaba dirigido a la población adolescente de la ciudad de Toluca, Estado de México. Docente de las materias de Seminario de Investigación Organizacional, Prácticas Organizacionales, Teorías del Conflicto y Teorías del Cambio en la Universidad Autónoma del Estado de Morelos, Facultad de Psicología. Actualmente realiza un Doctorado en Psicología Social y Ambiental, en donde adelanta el proyecto de tesis "Un Programa Hidrológico Sostenible". 